\title{
Altered plasma pharmacokinetics of ceftiofur hydrochloride in cows affected with severe clinical mastitis
}

\author{
P. J. Gorden, ${ }^{* 1}$ M. D. Kleinhenz, ${ }^{*}$ L. W. Wulf, ${ }^{*} \dagger$ B. KuKanich, $\ddagger$ C. J. Lee, ${ }^{*} \dagger^{2}$ C. Wang, ${ }^{*}$ and J. F. Coetzee ${ }^{*} \dagger^{1}$ \\ *Veterinary Diagnostic and Production Animal Medicine, and \\ †Pharmacology Analytical Support Team (PhAST), Veterinary Diagnostic Laboratory, College of Veterinary Medicine, lowa State University, \\ Ames 50011 \\ ‡Department of Anatomy and Physiology, College of Veterinary Medicine, Kansas State University, Manhattan 66502
}

\begin{abstract}
Mastitis is a frequent problem among dairy cows, reducing milk yield and increasing cull rates. Systemic therapy with the cephalosporin antimicrobial ceftiofur hydrochloride (CEF) may improve therapeutic outcomes, but the incidence of $\mathrm{CEF}$ violative residues has increased annually since 2011. One potential explanation is that disease status may alter the pharmacokinetics (PK) of CEF. To test this hypothesis, we compared the plasma $\mathrm{PK}$ of $\mathrm{CEF}$ in healthy cows with those with severe endotoxic mastitis. Eight cows with naturally occurring mastitis and 8 clinically healthy cows were treated with $2.2 \mathrm{mg}$ of CEF per kilogram of body weight once daily for $5 \mathrm{~d}$ via the intramuscular route. Blood was collected at $0,0.33,0.67,1,1.5,2,3,4,8,16$, and $24 \mathrm{~h}$ after the first CEF administration and every $8 \mathrm{~h}$ thereafter until $120 \mathrm{~h}$ after the final dose. Plasma samples were analyzed for CEF concentrations using liquid chromatography coupled with mass spectrometry. With the exception of time 0, CEF was detected at all time points. The disease group had a significantly higher plasma CEF concentration at $t=3 \mathrm{~h}$ after the first injection and a significantly lower plasma concentration from 40 to $152 \mathrm{~h}$ following the first injection, with the exception of the $t=64 \mathrm{~h}$ time point. Data following the first injection (time $0-24 \mathrm{~h}$ ) were fit to a single-dose, noncompartmental PK model. This model indicated that the disease group had a shorter plasma half-life. A multidose, noncompartmental model was used to determine steady-state PK. Compared with control cows, the disease group had an initially higher peak concentration and a higher volume of distribution
\end{abstract}

Received August 10, 2015

Accepted October 1, 2015.

${ }^{1}$ Corresponding authors: pgorden@iastate.edu and hcoetzee@ iastate.edu

${ }^{2}$ Current address: Division of Food and Environmental Sciences, Wonkwang University, Iksan, Jeonbuk, South Korea. and drug clearance rates. The disease group also had a lower area under the curve per dosing interval, steadystate concentration maximum, and dose-adjusted peak steady-state concentration. All other PK parameters were not different between the 2 groups. Altered PK, as suggested by this trial, may contribute to an increased risk for the development of a violative residue in meat. Further research is needed to more completely characterize drug distribution in diseased cattle and to study the effect of coadministration of other drugs on drug distribution.

Key words: bovine mastitis, ceftiofur, pharmacokinetics, drug residue

\section{INTRODUCTION}

Mastitis is the most common reason for therapeutic antimicrobial use in dairy cattle, according to the 2007 USDA National Animal Health Monitoring Survey. This survey of the dairy industry in the United States indicated that $18.2 \%$ of respondents' cows had been treated for mastitis during the previous 12 mo (USDA, APHIS, 2008). Coliforms are the most common bacterial group causing clinical mastitis, and these infections generally result in more severe infections, with decreased survivability, than other pathogens (Wenz et al., 1998; Erskine et al., 2002; Oliveira et al., 2013).

Reasons for decreased survivability following mastitis include the development of endotoxic conditions with severe intramammary (IMM) tissue damage and, potentially, the development of secondary disease. Secondary bacteremia develops in $45 \%$ of cows with severe mastitis (Wenz et al., 1998). Systemic ceftiofur (CEF) treatment can potentially address severe mastitis and secondary infections. When treated with CEF, fewer cows were sold or died as a result of severe coliform mastitis as compared with cows that were not treated (Erskine et al., 2002). Ceftiofur is a third-generation cephalosporin that is appropriate for use against coliform infections if effective concentrations can be achieved and maintained at the infection site. As a 
result, systemic treatment with $\mathrm{CEF}$, with or without IMM therapy, has been included in many veterinary treatment protocols for moderate or severe clinical mastitis.

Over the past several years, concerns about veterinary drug use have increased, especially around the effect on antimicrobial efficacy in humans and the presence of drug residues in milk and meat. Since fiscal year 2011, violative CEF residues in culled dairy cows from inspector-generated samples have increased more than 5-fold (USDA, FSIS, OPHS, 2013a,b; USDA, FSIS, OPHS, 2014a,b). In 2012 the US Food and Drug Administration (FDA) issued a prohibition against extra-label drug use of cephalosporins in bovines (US FDA, 2012). Given the prohibition of a drug so important to the dairy industry, there is clearly a significant regulatory concern about the increase in violative residues. Although the cause of increasing CEF residues is likely multifactorial, one potential explanation may be altered pharmacokinetics (PK) and residue depletion of CEF in diseased dairy cattle.

During the drug approval process, sponsoring companies must present the FDA Center for Veterinary Medicine with toxicological and residue depletion studies. Based on these data, the FDA Center for Veterinary Medicine establishes withdrawal periods for meat and milk, if approved for lactating dairy cattle. However, these studies are performed on healthy animals, not animals suffering from infectious diseases (US FDA, 2006a). Because antimicrobials are not intended for healthy animals, data on drug metabolism in diseased animals would provide veterinarians with evidence to more accurately prescribe veterinary drugs and also to better predict residue depletion in these diseased animals. In turn, this could improve treatment efficacy and reduce the risk for violative residues in marketed animals. However, few data from cattle available in the veterinary literature address this topic. The objective of this study was to compare the plasma PK of CEF between healthy dairy cattle and those afflicted with severe clinical mastitis. Our hypothesis was that cows affected with severe infectious disease would have altered CEF PK relative to healthy cows, necessitating variance in dose regimens and withdrawal periods.

\section{MATERIALS AND METHODS}

\section{Animals and Eligibility Criteria}

This study was completed at the Iowa State University Dairy Farm. The Iowa State University lactating herd consists of approximately 400 animals (approximately $90 \%$ Holstein and $10 \%$ Jersey), with 365-d rolling herd averages of $11,324 \mathrm{~kg}$ of milk, $415 \mathrm{~kg}$ of fat, and $357 \mathrm{~kg}$ of protein. Throughout the trial, cows were housed in a free-stall barn bedded with recycled manure solids, which is standard practice for this dairy. Cows were fed a TMR and watered ad libitum. Cows were milked 3 times daily at 4 a.m., 12 p.m., and 8 p.m. Cow housing and management met or exceeded the recommendations listed in the Guide for Care and Use of Agricultural Animals in Research and Teaching (FASS, 2010). The herd was vaccinated with a J5 core antigen vaccine 42 and $28 \mathrm{~d}$ before calving and again 25 and $90 \mathrm{~d}$ following calving. The Iowa State University Institutional Animal Use and Care Committee approved the research protocol before commencement of trial procedures (protocol number 6-14-7820-B).

Eight cows that presented with naturally occurring, acute severe toxic clinical mastitis, as described by Wenz et al. (2001), were selected to participate in the trial (disease group). The disease group had a mean $( \pm \mathrm{SD})$ weight of $607 \pm 77 \mathrm{~kg}$ (range $453-689 \mathrm{~kg}$ ). The milking crew of the farm first identified each diseasegroup cow based on an acute drop in milk production, abnormal milk, and severe swelling in the affected gland. At enrollment, all cows had evidence of systemic clinical signs associated with endotoxemia. To qualify, cows had to present with (1) at least one hot, swollen quarter secreting abnormal milk; (2) an acute decrease in milk production; and (3) evidence of systemic disease involvement determined by the presence of an elevated rectal temperature, depression, dehydration, anorexia, and decreased blood circulation as determined by the presence of congested mucous membranes or delayed capillary refill time.

Upon identification of a disease cow with severe mastitis, a healthy herd mate was matched by breed, DIM, and lactation number to serve as a control. The control group's mean $( \pm \mathrm{SD})$ weight was $650 \pm 100 \mathrm{~kg}$ (range 462-751 kg). Disease and control cows were eligible for the trial if they had not been treated with systemic or IMM CEF within the past $20 \mathrm{~d}$ and were healthy before enrollment. Furthermore, the cows needed to be 10 or more days from their next scheduled dry period.

\section{Study Design}

Disease and control cows were enrolled immediately following the noon milking. Throughout the trial, cows were milked by trial personnel per the farm milking protocol. Prior to the noon milking for the disease-group cows, a milk sample was aseptically collected from the mastitic quarter for bacterial culture. This sample was kept on ice until it was transferred to the laboratory for microbiological analysis. 
After milking, disease and control cows were weighed and placed in treatment chutes; two 10-mL blood samples were collected from the jugular vein in blood tubes containing freeze-dried heparin for plasma harvest (Becton, Dickinson and Co., Franklin Lakes, $\mathrm{NJ}$ ). Disease and control cows then received $2.2 \mathrm{mg}$ of CEF equivalents per kilogram of $\mathrm{BW}$ in the form of CEF hydrochloride (Excenel RTU EZ, Zoetis, Florham Park, NJ) via i.m. injection on the left side of the neck. Injections were given on alternating sides of the neck each day. In accordance with the manufacturer's recommendations, injection volumes were divided so that no more than $10 \mathrm{~mL}$ was administered per injection site. The CEF hydrochloride injections were repeated at approximately 24 -h intervals for $5 \mathrm{~d}$. At the discretion of the treating veterinarian, ancillary therapies of flunixin meglumine (FLU; $2.2 \mathrm{mg} / \mathrm{kg}$ i.v.; Prevail, VetOne, Boise, ID), $7.2 \%$ hypertonic saline $(3-5 \mathrm{~mL} /$ $\mathrm{kg}$ i.v.; Hypertonic Saline 7.2\%, VetOne), and oral fluids (20-40 L) were also provided for the disease group per standard treatment protocols in place for the dairy farm, as needed. The oral fluids consisted of tap water with $0.5 \mathrm{~kg}$ of an oral electrolyte powder (Fresh Cow Drench, IBA Inc., Millbury, MA) and $0.5 \mathrm{~kg}$ of dehydrated alfalfa meal added. The control cows received no ancillary therapy.

According to the farm treatment protocols, IMM therapy was based on the results of bacterial culture. For cows with gram-negative mastitis, negative culture results, or agents determined to be nonresponsive to IMM therapy (e.g., Prototheca zopfii or yeast), no IMM therapy was to be used. If a gram-positive agent was identified, IMM tubes that did not contain CEF were to be used according to label directions.

Blood samples were collected using venipuncture from the jugular vein into heparinized tubes at $0.33,0.67,1$, $1.5,2,3,4,8,16$, and $24 \mathrm{~h}$ after the first dose of CEF and approximately every $8 \mathrm{~h}$ thereafter, concluding 120 $\mathrm{h}$ after the last dose. After blood was collected, samples were immediately placed on ice until plasma could be harvested. Within one hour of collection, blood samples were centrifuged for $10 \mathrm{~min}$ at $1,000 \times g$ at $20^{\circ} \mathrm{C}$, and 5 $\mathrm{mL}$ of plasma was collected and frozen at $-70^{\circ} \mathrm{C}$ until it was analyzed for CEF plasma concentration.

Milk samples were cultured on brain heart infusion agar containing 5\% sheep blood and MacConkey Agar (Remel Microbiology Products, Lenexa, KS) using sterile, cotton-tipped swabs. Plates were inverted, incubated at $37^{\circ} \mathrm{C}$, and evaluated after 24 and $48 \mathrm{~h}$ for bacterial growth. Bacteria were identified based on visual appraisal of the plates using typical colony characteristics as described by the National Mastitis Council (1999).

\section{Plasma CEF Concentration Analysis}

Plasma concentrations of CEF were determined using liquid chromatography coupled with mass spectrometry. The liquid chromatography-mass spectrometry system consisted of an Agilent 1100 pump, autosampler, and column compartment (Agilent Technologies, Santa Clara, CA) coupled to an ion trap mass spectrometer (LTQ, Thermo Scientific, San Jose, CA). Total CEF concentrations (expressed as CEF equivalents) were determined by cleavage of $\mathrm{CEF}$, its metabolites, and protein-bound residues to desfuroylceftiofur using dithioerythritol, followed by derivatization with iodoacetamide. The resulting stable derivative, desfuroylceftiofur acetamide (DCA), was then analyzed by liquid chromatography-mass spectrometry. The internal standard was deuterated CEF (d3-CEF), which became d3-DCA upon cleavage and derivatization.

Cleanup of the derivatized samples was performed by solid-phase extraction using Oasis HLB cartridges (Waters Corp., Milford, MA). Plasma samples, plasma calibrators, and quality-control samples $(200 \mu \mathrm{L})$ were treated with $3 \mathrm{~mL}$ of $0.5 \%$ dithioerythritol in borate buffer $(0.05 N, \mathrm{pH} 9.0)$ after addition of $10 \mu \mathrm{L}$ of a 10 $\mathrm{ng} / \mu \mathrm{L}$ solution of the internal standard, d3-CEF. The samples were then vortexed for $5 \mathrm{~s}$ and placed in a $50^{\circ} \mathrm{C}$ water bath for $15 \mathrm{~min}$. Upon removal from the water bath and cooling to room temperature, $0.5 \mathrm{~mL}$ of $14 \%$ iodoacetamide in phosphate buffer $(0.025 \mathrm{M}, \mathrm{pH} 7)$ was added, and the samples were left in the dark for $30 \mathrm{~min}$.

Following derivatization, the samples were cleaned on an Oasis HLB solid-phase extraction column $(60 \mathrm{mg} / 3$ $\mathrm{mL}$ ) that was preconditioned with $1 \mathrm{~mL}$ of methanol followed by $1 \mathrm{~mL}$ of water. The sample was then transferred to the solid-phase extraction column and allowed to pass slowly through the HLB column. The column was washed with a $1-\mathrm{mL}$ portion of $5 \%$ (vol/vol) solution of methanol in water and then dried for 5 min with a flow of nitrogen. Elution of the derivatized samples was then performed with two $0.75-\mathrm{mL}$ portions of $5 \%$ (vol/vol) acetic acid in acetonitrile. The eluate was dried at $50^{\circ} \mathrm{C}$ with a stream of nitrogen in a Turbovap evaporator (Biotage, Charlotte, NC).

The dry residue was then reconstituted with $100 \mu \mathrm{L}$ of $25 \%$ ( $\mathrm{vol} / \mathrm{vol}$ ) acetonitrile in water and vortexed and then diluted with $50 \mu \mathrm{L}$ of water and vortexed again. The tube contents were transferred to an autosampler vial fitted with a glass insert, and the injection volume was set to $15 \mu \mathrm{L}$. The mobile phases consisted of (A) $0.1 \%$ formic acid in water and (B) $0.1 \%$ formic acid in acetonitrile at a flow rate of $0.25 \mathrm{~mL} / \mathrm{min}$. The mobile phase began at $10 \% \mathrm{~B}$ with a linear gradient to $95 \% \mathrm{~B}$ at $8 \mathrm{~min}$, which was maintained for $1.5 \mathrm{~min}$, followed 
by reequilibration to $10 \%$ B. Separation was achieved with an ACE C18 column (ACE $3 \mathrm{C} 18,150 \mathrm{~mm} \times$ $2.1 \mathrm{~mm}, 3-\mu \mathrm{m}$ particles, Mac-Mod Analytical, Chadds Ford, PA) maintained at $40^{\circ} \mathrm{C}$. The DCA was eluted at $3.70 \mathrm{~min}$, and the internal standard, d3-DCA, was eluted at $3.66 \mathrm{~min}$.

Sequences consisting of plasma blanks, calibration spikes, quality-control samples, and bovine plasma samples were then batch processed with an automated processing method developed in the Xcalibur software (Thermo Scientific), which identified and integrated each sample peak. The calibration curve was calculated based on a weighted $(1 / \mathrm{X})$, linear fit. Plasma concentrations of CEF in trial samples were calculated based on this calibration curve. Results were then viewed in the Quan Browser portion of the Xcalibur software. Calibration spikes were used from 1 to $10,000 \mathrm{ng} / \mathrm{mL}$. The standard curve had a linear range from 1 to 2,000 $\mathrm{ng} / \mathrm{mL}$ with a correlation coefficient of 0.998 . All standards were within $\pm 10 \%$ of the nominal value in this range. A quadratic fit was needed to fit in the last 2 calibrators at 5,000 and $10,000 \mathrm{ng} / \mathrm{mL}$. The limit of detection was $0.4 \mathrm{ng} / \mathrm{mL}$, and the limit of quantification was $2 \mathrm{ng} / \mathrm{mL}$.

\section{PK Analysis}

Because of the trial design, the PK data could not be modeled using only one model. Therefore, both singledose and multidose PK analyses were performed with computer software using noncompartmental methods (Phoenix WinNonlin, Certara L.P., Princeton, NJ). For the single-dose PK analysis, the maximum plasma concentration $(\mathbf{C m a x})$ for the first dose $\left(\mathbf{C m a x}_{1 \text { st dose }}\right)$, time to $\mathrm{Cmax}\left(\boldsymbol{T}_{\max } \mathbf{1 s t}_{\text {dose }}\right)$, and area under the curve from 0 to $24 \mathrm{~h}$ among all cows were determined directly from plasma samples collected after the first dose of CEF until the second dose was administered. The terminal half-life of the first dose $\left(\mathbf{T} \mathbf{1} / \mathbf{2}_{1 \text { st dose }}\right)$ was determined from at least 3 time points on the terminal portion of the plasma profile before the second dose was administered.

To complete the multidose model, time points $(\boldsymbol{t})$ that were equally distributed were used, starting with $t=8$ $\mathrm{h}$ and continuing with the time points every $8 \mathrm{~h}$ through the end of the trial. The Cmax at steady state per dose administered was determined by dividing steady-state Cmax by the actual dose administered. The terminal half-life following the last dose was determined from at least 6 time points on the terminal portion of the plasma profile after the last dose. The area under the curve at steady state for the dosing interval $\left(\mathbf{A} \mathbf{U} \mathbf{C}_{\text {tau }}\right)$ was determined directly from the data at 96 to $120 \mathrm{~h}$ after the first dose of CEF. The volume of distribution (area method) per fraction of the dose absorbed $\left(\boldsymbol{V}_{z} / \boldsymbol{F}\right)$ was calculated with the following equation:

$$
\text { Dose } /\left(\lambda z \times \mathrm{AUC}_{\text {tau }}\right),
$$

where $\lambda z$ represents the individual estimate of the terminal elimination rate constant, which is calculated by determining the log-linear regression of the terminal portions of the plasma concentration versus the time curves. The plasma clearance per fraction of the dose absorbed at steady state $(\mathbf{C l} / \boldsymbol{F})$ was calculated with the following equation:

$$
\text { Dose/AUC } \mathrm{Aau}_{\text {. }} \text {. }
$$

The accumulation ratio was determined by dividing $\mathrm{AUC}_{\text {tau }}$ by $\mathrm{AUC}$ extrapolated to infinity after the last dose. The mean residence time was calculated by dividing the area under the moment curve extrapolated to infinity after the last dose by the AUC extrapolated to infinity after the last dose.

\section{Data Analysis}

Statistical analysis was performed using SAS 9.4 (SAS Institute Inc., Cary, NC). All PK data are reported as geometric means. Disease and control groups were compared using the Wilcoxon 2-sample rank-sum test (Mann-Whitney test). Multiple variables (i.e., drug concentrations and PK values) had distributions that were right skewed with long tails. Therefore, nonparametric methods were used to analyze the data in this situation. Statistical significance was established when $P<0.05$.

\section{RESULTS}

Eight cows (7 Holstein, 1 Jersey) were enrolled in the trial as disease-group animals. At enrollment, diseasegroup cows ranged from 2 to 5 lactations and 2 to 354 DIM. All cultures from mastitis samples were pure cultures and identified as either Escherichia coli $(\mathrm{n}=$ $5)$ or Klebsiella spp. $(\mathrm{n}=3)$. Following the 10 -d trial period, 5 of the 8 disease-group cows were immediately culled from the herd and 1 additional cow was culled within $60 \mathrm{~d}$; all culls resulted from the clinical mastitis episode that initiated their enrollment in the trial. The remaining 2 cows, 1 with mastitis caused by $E$. coli and 1 with Klebsiella spp., remained in the herd for at least $60 \mathrm{~d}$ following the clinical episode of mastitis. No comparative parameters statistically differed between disease and control cows (Table 1). 
Table 1. Mean \pm SD DIM, lactation number, age, cow weight, and milk production for 8 severe mastitis cows (disease group) and 8 control cows

\begin{tabular}{lcc}
\hline Parameter & Disease group & Control group \\
\hline DIM & $119.2 \pm 122.6$ & $119.5 \pm 125.6$ \\
Lactation number & $3.4 \pm 0.9$ & $3.1 \pm 1.0$ \\
Age of cow (yr) & $5.0 \pm 1.4$ & $4.6 \pm 1.3$ \\
Cow weight (kg) & $607 \pm 77$ & $650 \pm 100$ \\
305-d mature-equivalent milk production $(\mathrm{kg})$ & $10,246 \pm 1,118$ & $9,996 \pm 1,352$ \\
\hline
\end{tabular}

Seven of the eight disease-group cows received ancillary therapy of FLU for an average of $2.3 \mathrm{~d}$ (range 1-5), 5 of the 8 received hypertonic saline an average of 1.2 d (range 1-2), and all 8 received oral fluids an average of 3.9 d (range 2-9). None of the cows received IMM antimicrobial therapy.

No cow had detectable plasma CEF at enrollment (time 0), and $\mathrm{CEF}$ was detected in all subsequent samples throughout the entire study period. The CEF concentrations were significantly higher in the disease group $3 \mathrm{~h}$ after the first injection and then significantly lower at 40,48, 56, 72, 80, 88, 96, 104, 112, 120, 128, 136, 144, and $152 \mathrm{~h}$ after the first injection (Figures 1 and 2).

Several PK parameters differed between disease and control cows. The results of the single-dose PK model are displayed in Table 2, and those from the multidose model are displayed in Table 3. Compared with control cows, the single-dose PK model indicated a shorter T1 $/ 2_{1 \text { st dose }}(P=0.038)$ for the disease group. The multidose PK model indicated that the disease group had a higher $V z / F(P=0.001)$ and $\mathrm{Cl} / F(P=0.0006)$ com-

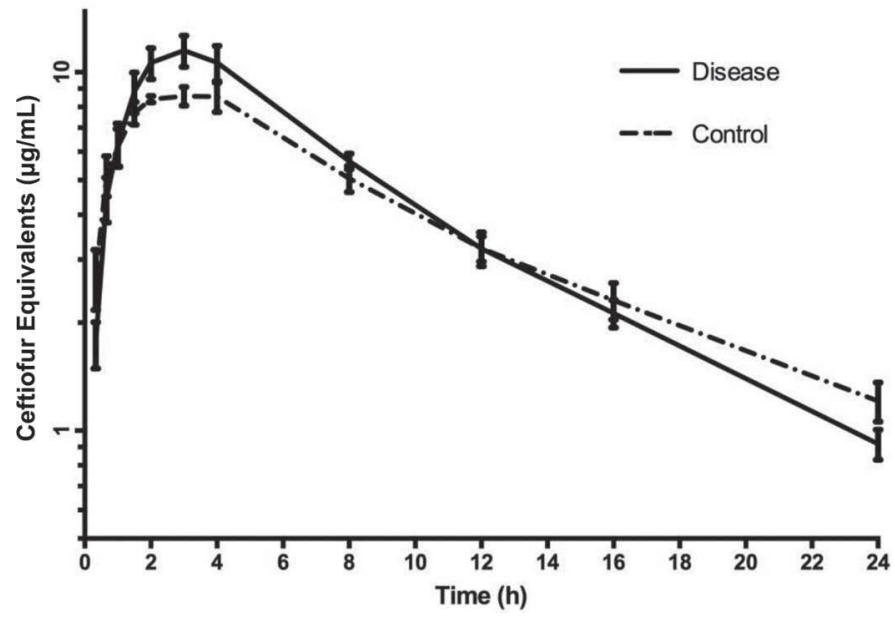

Figure 1. Log transformations of mean plasma ceftiofur equivalents concentration $( \pm 1 \mathrm{SE})$ for 8 disease-group cows with severe mastitis and 8 healthy control cows following a single administration of ceftiofur hydrochloride at $2.2 \mathrm{mg}$ of ceftiofur equivalents per kilogram of BW. pared with the control group. The disease group had lower $\operatorname{AUC}_{\text {tau }}(P=0.0006)$, steady-state $\operatorname{Cmax}(P=$ $0.0006)$, and steady-state Cmax per dose administered $(P=0.0006)$. There was also a tendency for a longer $\mathrm{T} 1 / 2_{\text {last dose }}$ in the disease group $(P=0.065)$. All other PK parameters were not different between the 2 groups in both models.

\section{DISCUSSION}

Ceftiofur is a third-generation cephalosporin marketed for use in multiple food-animal species. In the dairy industry, CEF is marketed for systemic and IMM use. Based on our experience and that of others (Zwald et al., 2004; Sawant et al., 2005), CEF is likely the most commonly used antimicrobial in the United States dairy industry due to its broad spectrum of activity, multiple formulations, and short milk and meat withholding periods. Within an hour of injection with CEF products, the parent compound is almost completely metabolized by the liver to desfuroylceftiofur and small concentrations of other nonpolar and polar metabolites (Jaglan et al., 1989; Brown et al., 1991). Desfuroylceftiofur has an exposed sulfhydryl group that forms reversible disulfide bonds with other sulfurous compounds, such as sulfhydryl-containing proteins and glutathione. These protein-bound CEF metabolites are not biologically active but can dissociate quickly in reduced environments to form active metabolites (Clarke et al., 1996). In cattle, protein binding is age dependent, with low levels in calves, whereas healthy adult cows have CEF metabolites that are $>90 \%$ bound (Brown et al., 1991). Repeated dosing with CEF may result in saturation of protein-binding sites, resulting in the presence of more unbound, active metabolites over time without increasing T1/2 (Halstead et al., 1992). More than 95\% of administered CEF sodium is excreted within $24 \mathrm{~h}$, with 61 to $77 \%$ of the total drug excreted through urine and the balance excreted in feces (Brown et al., 1991). Drug approval studies show that metabolism and excretion of CEF hydrochloride by cattle is similar to that of $\mathrm{CEF}$ sodium (US FDA, 1998), and tissue distribution of CEF is similar regardless of the CEF salt administered (US FDA, 1998; KuKanich et al., 2005). 
Table 2. Single-dose plasma pharmacokinetic parameters of ceftiofur equivalents after the first dose for disease and control cows using a multidose regimen ${ }^{1}$

\begin{tabular}{lcc}
\hline Parameter $^{2}$ & Disease group & \\
\hline Cmax $_{1 \text { st dose }}(\mu \mathrm{g} / \mathrm{mL})$ & $11.75(7.73-17.80)$ & Control group \\
$\operatorname{Tmax}_{1 \text { st dose }}(\mathrm{h})$ & $2.54(2-4)$ & $9.58(7.73-13.26)$ \\
$\mathrm{T} 1 / 2_{1 \text { st dose }}(\mathrm{h})$ & $6.57(5.04-8.04)$ & $2.87(2-4)$ \\
$\mathrm{AUC}_{0-24}(\mathrm{~h} \times \mu \mathrm{g} / \mathrm{mL})$ & $106.4(85.94-124.54)$ & $9.13(6.59-9.80)^{*}$ \\
\hline
\end{tabular}

${ }^{1}$ Results are presented as geometric mean for ceftiofur equivalents. Values in parentheses are the range.

${ }^{2} \mathrm{Cmax}_{1 \text { st dose }}=$ maximum plasma concentration for the first dose; $\operatorname{Tmax}_{1 \text { st dose }}=$ time to $\mathrm{Cmax}$; $\mathrm{T} 1 / 2_{1 \text { st dose }}=$ terminal half-life of the first dose; $\mathrm{AUC}_{0-24}=$ area under the concentration curve from 0 to $24 \mathrm{~h}$.

${ }^{3}$ Cows with severe toxic mastitis.

${ }^{*}$ Geometric means within the columns differ $(P<0.05)$.

Over the last several years, concerns about veterinary drug use, its effect on antimicrobial efficacy in humans, and the presence of drug residues in milk and meat have increased. This is especially true for medically important drugs, including cephalosporins (US FDA, 2006b). In 2012 the FDA issued a prohibition against extra-label drug use of cephalosporins in bovines for those cephalosporins with analogs in human medicine (US FDA, 2012). Although mastitis is an extra-label condition for all systemic CEF products, the FDA does not restrict extra-label conditions as long as the dose, duration, and route-of-administration label conditions are followed (US FDA, 2012). Using published literature (e.g., Wenz et al., 1998; Erskine et al., 2002), veterinarians can justify this extra-label therapy provided it is in compliance with the requirements of the Animal Drug Use Clarification Act (US FDA, 1996).

In the dairy industry, CEF is primarily affected by the FDA prohibition; however, CEF residues have increased substantially since fiscal year 2011. Between fiscal year 2011 and 2012, confirmed violative residues from inspector-generated samples in culled dairy cattle increased from 53 to 130 (USDA, FSIS, OPHS, 2013a, 2014a), rising to 283 in fiscal year 2014 (USDA, 2014b). These reports indicate that $\mathrm{CEF}$ residues are now the most common violative residues found in cull dairy

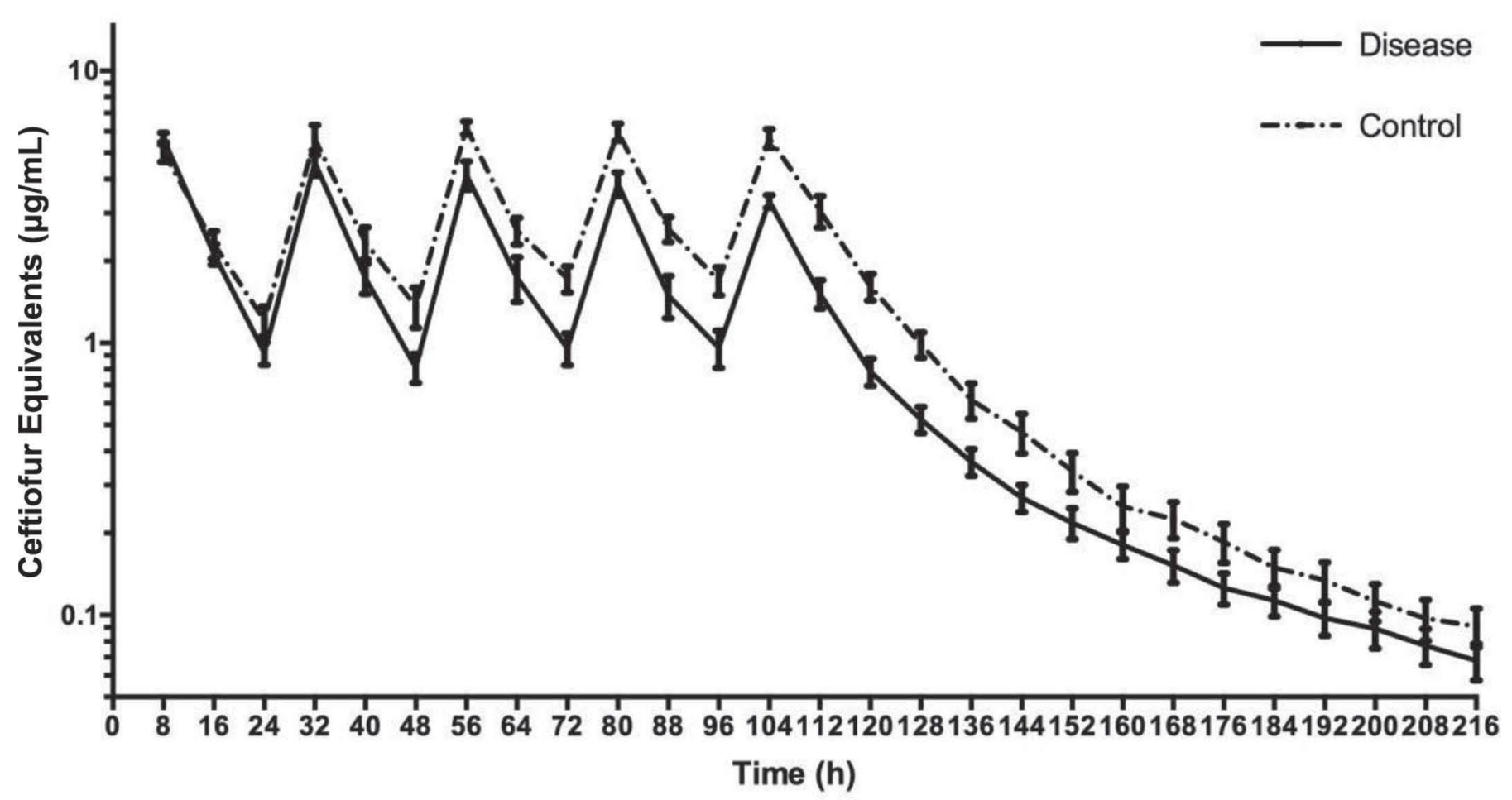

Figure 2. Log transformations of mean plasma ceftiofur equivalents concentration ( $\pm 1 \mathrm{SE}$ ) for 8 disease-group cows with severe mastitis and 8 healthy control cows following 5 administrations of ceftiofur hydrochloride at $2.2 \mathrm{mg}$ of ceftiofur equivalents per kilogram of BW. 
Table 3. Multidose plasma pharmacokinetic parameters of disease and control cows ${ }^{1}$

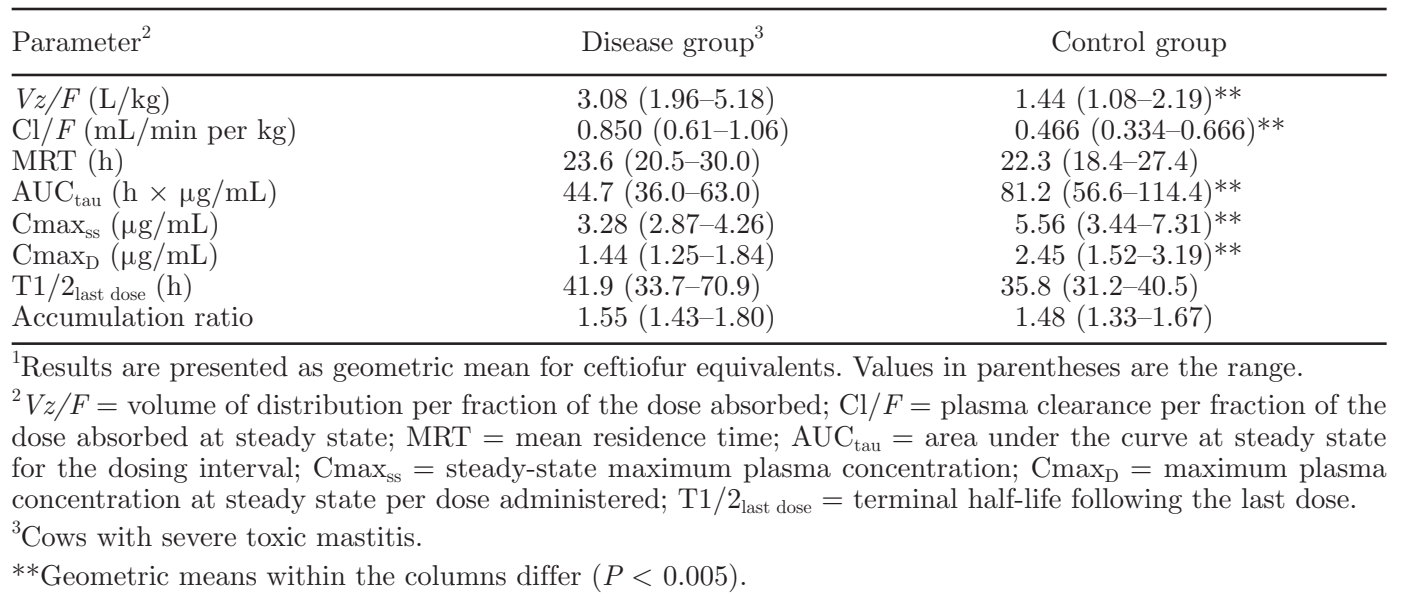

cattle. Given the extra-label prohibition and the importance of the drug to the dairy industry, the concern is extreme about this level of violative residues. Although the reason for this level of violative residues is likely multifactorial, one potential cause may be altered PK and residue depletion of CEF in diseased dairy cattle.

The objective of this trial was to determine whether plasma PK for CEF were different between healthy cows and cows with severe disease. Our findings suggest that disease may indeed alter the PK of CEF in diseased cattle, resulting in a shorter $\mathrm{T} 1 / 2_{1 s t}$ dose in the disease group when the data were modeled for a single dose. When multidose modeling was applied, $V z / F$ had more than a 2 -fold increase and plasma clearance rate had an almost 2-fold increase. Additionally, steadystate Cmax, steady-state Cmax per dose administered, and AUC were significantly lower in the disease group, resulting in more than a $40 \%$ reduction in each parameter. Following the first injection, our single PK data were fairly close for $\mathrm{Cmax}_{1 \mathrm{st} \text { dose }}$ and $\operatorname{Tmax}_{1 \mathrm{st} \text { dose }}$ compared with package-insert data supplied with the product (Zoetis Inc., 2013). However, the T1/2 $2_{1 s t}$ dose data were substantially shorter than the package insert ( $8.13 \mathrm{~h}$ for the control group vs. $29.3 \mathrm{~h}$ from the package insert). The likely explanation for this difference was the limited amount of time available $(t=3$ $\mathrm{h}$ to $t=24 \mathrm{~h}$ ) to follow the depletion curve after the $\operatorname{Cmax}_{1 \mathrm{st} \text { dose }}$ before the second dose was administered. During that time, the depletion curve was fairly linear, unlike the depletion curve that occurred following the fifth dose, leading to a much shorter depletion estimate for the single PK model (see Figure 1). In contrast, the $\mathrm{T} 1 / 2_{\text {last dose }}$ was much closer to the value reported in the package insert.

A weakness of the multidose PK model was that the first time point $(t=8 \mathrm{~h})$ to be included in this model occurred after the $\mathrm{Cmax}_{1 \mathrm{st} \text { dose }}$ and $\operatorname{Tmax}_{1 \mathrm{st} \text { dose }}$. This biased the reported value for $\mathrm{AUC}_{\text {tau }}$ lower. As a result, the values for $\mathrm{Vz} / \mathrm{F}$ and $\mathrm{Cl} / \mathrm{F}$ are overestimates in the data reported. However, because this occurred in both groups and $\mathrm{Cmax}_{1 \mathrm{st} \text { dose }}$ and $\mathrm{Tmax}_{1 \text { st dose }}$ were not statistically different, the changes should be proportional between the 2 groups, indicating that although the values are not exact, the change between the 2 groups for $\mathrm{AUC}_{\text {tau }}, V z / F$, and $\mathrm{Cl} / F$ are true. This can be verified by studying the concentration curves presented in Figure 2 and noting that a statistically significant change in concentrations started to occur at $t=40 \mathrm{~h}$ through $t=152 \mathrm{~h}$, with the exception of $t=64 \mathrm{~h}$.

Many physiological changes could explain the PK changes we found, including altered absorption of the drug because of altered blood flow to the injection site; reduced plasma protein levels in diseased animals that allowed unbound CEF to move more freely to tissue or be excreted; competition with coadministered drugs (e.g., FLU) for protein-binding sites; changes in membrane permeability in tissue, such as the mammary gland; altered liver and kidney function due to altered blood flow or altered enzyme systems (van Miert, 1990); or altered function of membrane-bound drug transporters (Petrovic et al., 2007).

Despite the widespread use of CEF in dairy cattle, there is a paucity of data comparing PK parameters of CEF between healthy and diseased cows. In one trial, Erskine et al. (1995) compared drug concentrations in healthy cows versus cows experimentally challenged with IMM E. coli; however, they used a different dosing strategy (3 mg/kg i.v. every $12 \mathrm{~h}$ for 3 treatments), which resulted in all drug administration occurring within $24 \mathrm{~h}$. In accordance with our findings in the single-dose PK model, they reported no difference in peak concentrations of $\mathrm{CEF}$ in the serum of treated versus healthy control cows. In their trial, they found higher serum concentrations of active CEF metabolites 
in the control group, suggesting that diseased cattle had increased distribution out of the plasma pool. We found a similar increase in plasma distribution of drug, but we did not measure active compound. However, in contrast to our results, they reported peak serum concentrations $90 \%$ less than the values measured in our trial. This disparity may be due to methodological differences; their assay did not account for protein-bound metabolites, which could make CEF concentrations appear low.

Other researchers have compared CEF concentrations using both HPLC and microbiological assay in feedlot cattle implanted with tissue chambers; half of the chambers were later inoculated with Mannheimia haemolytica. Both methodologies indicate that total DCA is significantly higher in infected than uninfected tissue chambers, even within a single animal. Interestingly, the ratio of active CEF to total DCA was higher in the infected chamber than uninfected chamber (Clarke et al., 1996). Thus, it appears that a higher concentration of total CEF (both bound and active) accumulates at the site of infection by passively moving through disrupted endothelial cell barriers and from the binding of CEF to acute phase proteins, such as $\alpha_{1}$-anti-trypsin, which rapidly move to sites of infection (Walker et al., 1994; Clarke et al., 1996). Outward fluxing membrane transporter pumps could also be impaired because of inflammation (Petrovic et al., 2007). Movement through impaired membranes of the mammary gland may explain the higher $V z / F$ in diseased cattle described in our study.

Other comparisons of antimicrobial PK in diseased versus healthy animals have been completed in swine (Tantituvanont et al., 2009; Day et al., 2015) and chickens (Amer et al., 1998). The swine trials included pigs experimentally infected with porcine reproductive and respiratory syndrome virus (Tantituvanont et al., 2009) and pigs coinfected with porcine reproductive and respiratory syndrome virus and Streptococcus suis (Day et al., 2015), both of which were compared with healthy control animals. In these trials, volume of distribution and $\mathrm{Cl}$ were higher and plasma concentration values lower in diseased than healthy animals (Tantituvanont et al., 2009; Day et al., 2015). In the poultry trial, one group of healthy chickens was exposed to aflatoxin in their diet, and the control group was fed a diet devoid of aflatoxin. Both groups were treated with CEF via the i.v., i.m., or oral route. In the chickens treated via the oral and i.v. route, tissue concentrations of CEF were lower and CEF was eliminated more quickly (Amer et al., 1998). Taken together, these 3 trials (Amer et al., 1998; Tantituvanont et al., 2009; Day et al., 2015), combined with our work, suggest that more research on drug disposition is needed in disease-challenged animals. Future trials for drug approval should investigate the effect of disease when establishing therapeutic dosages and residue depletion time.

In this trial, our goal was to approximate treatment strategies used on commercial dairy farms. Therefore, research personnel did not interfere with treatment decisions regarding coadministered therapies. Administration of CEF to cows with moderate or severe mastitis reduces culling as a result of the mastitis event (Erskine et al., 2002). It is therefore included in treatment protocols for mastitis by many veterinarians. Flunixin meglumine is commonly included in the treatment of moderate to severe clinical mastitis (Kissell et al., 2015), despite inconsistent evidence of clinical benefits (Anderson et al., 1986; Dascanio et al., 1995; Green et al., 1997; Wagner and Apley, 2004; Yeiser et al., 2012). Admittedly, the coadministration of CEF and FLU potentially confounds the outcome. Flunixin meglumine is reported to be greater than $98 \%$ protein bound (Anderson et al., 1990; Odensvik and Johansson, 1995), which may actively compete with CEF for the same protein-binding sites. Although the PK changes that occur with the coadministration of CEF and FLU are not known, theoretically protein binding could be reduced for one or both drugs. If CEF was less protein bound as a result of coadministration of FLU, this could account for the changes that we noted for $V z / F$ and $\mathrm{Cl}$. However, in a pair of manuscripts, Whittem et al. $(1995,1996)$ reported no PK differences with CEF or aspirin following coadministration of single doses of each drug. Like FLU, aspirin is a nonsteroidal antiinflammatory drug that achieves high protein binding in the bovine (Lee, 2009). Additional research is needed to determine the effects of highly protein-bound drugs coadministered with other such drugs, including the coadministration of CEF and FLU, because this is how the drugs are typically used on dairy farms.

In conclusion, these data, coupled with those of other researchers, show that significant PK changes occur in diseased animals administered CEF relative to healthy, control animals. These outcomes potentially have public-health significance in that (1) drug efficacy could be lower than expected; (2) violative drug residues may increase in tissues of cull animals or milk; and (3) this may lead to increases in antimicrobial resistance. This body of evidence suggests that the drug approval process should be changed such that the physiological changes of health-challenged cows are addressed. It does not, however, lead to any conclusions regarding the outcomes of treating health-challenged cows nor the contribution of altered PK on the increasing violative CEF residues found in culled dairy cattle. Further work 
examining protein binding, tissue distribution, and tissue depletion of drugs and their influence on residue levels in diseased animals at the end of their withdrawal periods is necessary to more thoroughly characterize this problem.

\section{ACKNOWLEDGMENTS}

The authors thank Kelly Still-Brooks, DVM; Adlai Schuler; and Dan Breuer (from the Iowa State University College of Veterinary Medicine) for their assistance with sample collection. This trial was funded by Iowa State University's College of Veterinary Medicine (Ames). We also thank Gary Neubauer from Zoetis Inc. (Florham Park, NJ) for the donation of the ceftiofur hydrochloride used in this trial.

\section{REFERENCES}

Amer, A. M., E. M. Fahim, and R. K. Ibrahim. 1998. Effect of aflatoxicosis on the kinetic behaviour of ceftiofur in chickens. Res. Vet. Sci. 65:115-118.

Anderson, K. L., C. A. Neff-Davis, L. E. Davis, and V. D. Bass. 1990. Pharmacokinetics of flunixin meglumine in lactating cattle after single and multiple intramuscular and intravenous administrations. Am. J. Vet. Res. 51:1464-1467.

Anderson, K. L., A. R. Smith, R. D. Shanks, L. E. Davis, and B. K. Gustafsson. 1986. Efficacy of flunixin meglumine for the treatment of endotoxin-induced bovine mastitis. Am. J. Vet. Res. 47:13661372 .

Brown, S. A., P. S. Jaglan, and A. Banting. 1991. Ceftiofur sodium: disposition, protein-binding, metabolism, and residue depletion profile in various species. Acta Vet. Scand. Suppl. 87:97-99.

Clarke, C. R., S. A. Brown, R. N. Streeter, J. M. Clark, P. J. Hamlow, J. K. Callahan, V. L. Hubbard, A. K. Speedy, and G. E. Burrows. 1996. Penetration of parenterally administered ceftiofur into sterile vs Pasteurella haemolytica-infected tissue chambers in cattle. J. Vet. Pharmacol. Ther. 19:376-381.

Dascanio, J. J., G. D. Mechor, Y. T. Gröhn, D. G. Kenney, C. A. Booker, P. Thompson, C. L. Chiffelle, J. M. Musser, and L. D. Warnick. 1995. Effect of phenylbutazone and flunixin meglumine on acute toxic mastitis in dairy cows. Am. J. Vet. Res. 56:12131218.

Day, D. N., J. W. Sparks, L. A. Karriker, K. F. Stalder, L. W. Wulf, J. Zhang, J. Kinyon, M. Stock, R. Gehring, C. Wang, J. Ellingson, and J. H. Coetzee. 2015. Impact of an experimental PRRSV and Streptococcus suis co-infection on the pharmacokinetics of ceftiofur hydrochloride after intramuscular injection in pigs. J. Vet. Pharmacol. Ther. http://dx.doi.org/10.1111/jvp.12209.

Erskine, R. J., P. C. Bartlett, V. L. Van Lente, and C. R. Phipps. 2002. Efficacy of systemic ceftiofur as a therapy for severe clinical mastitis in dairy cattle. J. Dairy Sci. 85:2571-2575.

Erskine, R. J., R. C. Wilson, J. W. Tyler, K. A. McClure, R. S. Nelson, and H. J. Spears. 1995. Ceftiofur distribution in serum and milk from clinically normal cows and cows with experimental Escherichia coli-induced mastitis. Am. J. Vet. Res. 56:481-485.

FASS. 2010. Dairy cattle. Pages 74-88 in Guide for the Care and Use of Agricultural Animals in Teaching and Research. 3rd ed. FASS, Champaign, IL.

Green, M. J., L. E. Green, and P. J. Cripps. 1997. Comparison of fluid and flunixin meglumine therapy in combination and individually in the treatment of toxic mastitis. Vet. Rec. 140:149-152.

Halstead, S. L., R. D. Walker, J. C. Baker, R. E. Holland, G. E. Sytein, and J. G. Hauptman. 1992. Pharmacokinetic evaluation of ceftio- fur in serum, tissue chamber fluid, and bronchial secretions from healthy beef-bred calves. Can. J. Vet. Res. 56:269-274.

Jaglan, P. S., M. F. Kubicek, T. S. Arnold, B. L. Cox, R. H. Robins, D. B. Johnson, and T. J. Gilberson. 1989. Metabolism of ceftiofur. Nature of urinary and plasma metabolites in rats and cattle. J. Agric. Food Chem. 37:1112-1118.

Kissell, L. W., T. L. Leavens, R. E. Baynes, J. E. Riviere, and G. W. Smith. 2015. Comparison of pharmacokinetics and milk elimination of flunixin in healthy cows and cows with mastitis. J. Am. Vet. Med. Assoc. 246:118-125.

KuKanich, B., R. Gehring, A. I. Webb, A. L. Craigmill, and J. E. Riviere. 2005. Effect of formulation and route of administration on tissue residues and withdrawal times. J. Am. Vet. Med. Assoc. 227:1574-1577.

Lee, P. 2009. Analgesics, antiinflammatory, antipyretic drugs. Pages 457-492 in Veterinary Pharmacology and Therapeutics. 9th ed. J. E. Riviere and M. G. Papich, ed. Wiley-Blackwell, Ames, IA.

National Mastitis Council. 1999. Laboratory and Field Handbook on Bovine Mastitis. W. D. Hoard and Sons, Fort Atkinson, WI.

Odensvik, K., and I. M. Johansson. 1995. High-performance liquid chromatography method for determination of flunixin in bovine plasma and pharmacokinetics after single and repeated doses of the drug. Am. J. Vet. Res. 56:489-495.

Oliveira, L., C. Hulland, and P. L. Ruegg. 2013. Characterization of clinical mastitis occurring in cows on 50 large dairy herds in Wisconsin. J. Dairy Sci. 96:7538-7549.

Petrovic, V., S. Teng, and M. Piquette-Miller. 2007. Regulation of drug transporters during infection and inflammation. Mol. Interv. $7: 99-111$

Sawant, A. A., L. M. Sordillo, and B. M. Jayarao. 2005. A survey on antibiotic usage in dairy herds in Pennsylvania. J. Dairy Sci. 88:2991-2999.

Tantituvanont, A., W. Yimprasert, P. Werawatganone, and D. Nilubol. 2009. Pharmacokinetics of ceftiofur hydrochloride in pigs infected with porcine reproductive and respiratory syndrome virus. J. Antimicrob. Chemother. 63:369-373.

US FDA (US Food and Drug Administration). 1996. Extralabel drug use in animals. Fed. Regist. 61:57732-57746.

US FDA (US Food and Drug Administration). 1998. Freedom of Information Summary, Supplement to NADA 140-890, EXCENEL Sterile Suspension (ceftiofur hydrochloride injection). US Food Drug Admin., Washington, DC.

US FDA (US Food and Drug Administration). 2006a. General Principles for Evaluating the Safety of Compounds Used in FoodProducing Animals. Accessed Dec. 20, 2014. http://www.fda.gov/ ohrms/dockets/98fr/05d-0219-gdl0001.pdf.

US FDA (US Food and Drug Administration). 2006b. Guidance for Industry \#152: Evaluating the safety of antimicrobial new animal drugs with regard to their microbiological effects on bacteria of human health concern. Accessed Feb. 12, 2015. http://www.fda.gov/ downloads/AnimalVeterinary/GuidanceComplianceEnforcement/ GuidanceforIndustry/ucm052519.pdf.

US FDA (US Food and Drug Administration). 2012. New animal drugs; cephalosporin drugs; extralabel animal drug use; order of prohibition. Fed. Regist. 77:725-745.

USDA. APHIS (Animal and Plant Health Inspection Service). 2008 Reference of Dairy Cattle Health and Management Practices in the United States, National Animal Health Monitoring System, Dairy 2007, Part III. USDA, Washington, DC.

USDA, FSIS, OPHS (Food Safety and Inspection Service, Office of Public Health Science). 2013a. United States National Residue Program for Meat, Poultry, and Egg Products-2011 Residue Sample Results. Accessed Dec. 20, 2014. http://www.fsis.usda.gov/ wps/wcm/connect/f511ad0e-d148-4bec-95c7-22774e731f7c/2011_ Red_Book.pdf?MOD=AJPERES.

USDA, FSIS, OPHS (Food Safety and Inspection Service, Office of Public Health Science). 2014a. United States National Residue Program for Meat, Poultry, and Egg Products-2012 Residue Sample Results. Accessed Dec. 20, 2014. http://www.fsis.usda.gov/ wps/wcm/connect/be77fe0d-2295-417f-9472-6b43052068b9/2012 Red-Book.pdf?MOD=AJPERES. 
USDA, FSIS, OPHS (Food Safety and Inspection Service, Office of Public Health Science). 2013b. United States National Residue Program-2013 Residue Quarterly Reports. Accessed Dec. 20, 2014. http://www.fsis.usda.gov/wps/portal/fsis/topics/datacollection-and-reports/chemistry/residue-quarterly/ct_index.

USDA, FSIS, OPHS (Food Safety and Inspection Service, Office of Public Health Science). 2014b. United States National Residue Program-2014 Residue Quarterly Reports. Accessed Dec. 20, 2014. http://www.fsis.usda.gov/wps/portal/fsis/topics/datacollection-and-reports/chemistry/residue-quarterly/ct_index.

van Miert, A. 1990. Influence of febrile disease on the pharmacokinetics of veterinary drugs. Ann. Rech. Vet. 21(Suppl. 1):11S-28S.

Wagner, S. A., and M. D. Apley. 2004. Effects of two anti-inflammatory drugs on physiologic variables and milk production in cows with endotoxin-induced mastitis. Am. J. Vet. Res. 65:64-68.

Walker, J. L., C. R. Clarke, B. A. Lessley, and C. M. Hague. 1994. Effect of Pasteurella haemolytica infection on $\alpha_{1}$-acid glycoprotein and albumin concentrations in serum and subcutaneous tissue chamber fluid of calves. Res. Vet. Sci. 56:158-163.

Wenz, J. R., G. M. Barrington, F. B. Garry, R. P. Dinsmore, and R. J. Callan. 2001. Use of systemic disease signs to assess disease severity in dairy cows with acute coliform mastitis. J. Am. Vet. Med. Assoc. 218:567-572.
Wenz, J. R., G. M. Barrington, F. B. Garry, G. M. Goodell, and R. P. Dinsmore. 1998. Characterization of naturally occurring coliform mastitis. J. Dairy Sci. 81(Suppl. 1):38.

Whittem, T., D. A. Freeman, D. Hanlon, and K. Parton. 1995. The effects on the pharmacokinetics of intravenous ceftiofur sodium in dairy cattle of simultaneous intravenous acetyl salicylate (aspirin) or probenecid. J. Vet. Pharmacol. Ther. 18:61-67.

Whittem, T., D. A. Freeman, D. W. Hanlon, and K. Parton. 1996. The pharmacokinetics of salicylate in dairy cattle are not altered by simultaneous intravenous ceftiofur sodium and DL-lysine-acetyl salicylate (aspirin). J. Vet. Pharmacol. Ther. 19:104-108.

Yeiser, E. E., K. E. Leslie, M. L. McGilliard, and C. S. Petersson-Wolfe. 2012. The effects of experimentally induced Escherichia coli mastitis and flunixin meglumine administration on activity measures, feed intake, and milk parameters. J. Dairy Sci. 95:4939-4949.

Zoetis Inc. 2013. Excenel RTU EZ package insert. Accessed Jul. 21, 2015. https://www.zoetisus.com/_locale-assets/mcm-portalassets/products/pdf/exc13027_excenel_rtu_ez_brochure.pdf.

Zwald, A. G., P. L. Ruegg, J. B. Kaneene, J. D. Warnick, S. J. Wells, C. Fossler, and L. W. Halbert. 2004. Management practices and reported antimicrobial usage on conventional and organic dairy farms. J. Dairy Sci. 87:191-201. 